\title{
Calificación riesgo país y flujos de capital en México: 1998-2012
}

\author{
MARio Alberto Rosas Chimal a , Miguel Flores ORTEgA ${ }^{\text {b }}$ \\ a Universidad del Istmo, Facultad de Ciencias Empresariales, Carretera Chihuitan Ixtepec S/N , \\ 70110 Ixtepec, Oaxaca, México. E-mail: malroch1114@msn.com \\ b Instituto Politécnico Nacional, Sección de Estudios de Posgrado e Investigación, Av. Periférico \\ Sur No. 4863, Col. Ampliación Tepepan, Delegación Tlalpan, 16020, CDMX México. E-mail: \\ mfo@prodigy.net.mx
}

\begin{abstract}
RESUMEN
Este documento presenta evidencia empírica de la existencia de integración entre el índice de riesgo país caracterizado por el EMBI (Índice del Mercado de Bonos de los Países Emergentes) y el flujo de inversión extranjera de cartera hacia la economía mexicana. Se realiza la prueba de estacionariedad de Elliott, Rothenberg y Stock (1996) denominada ERS/GLS, que se complementa con la prueba M/GLS propuesta por Ng-Perron (2001). Se analizan conjuntamente las series temporales seleccionadas mediante la metodología VAR, lo que permitió encontrar evidencia de la existencia de una relación de largo plazo entre los flujos de inversión extranjera de cartera hacia México y el Índice EMBI.

Palabras clave: Modelos de series de tiempo, cointegración, raíz unitaria, VAR, mercados financieros.
\end{abstract}

\section{Country risk rating and capital flows in Mexico: 1998-2012}

\begin{abstract}
This paper presents empirical evidence of the existence of integration between country risk index EMBI(Emerging Market Bond Index) and the flow of foreign portfolio investment to the Mexican economy. Stationarity test Elliott, Rothenberg and Stock (1996) called ERS/GLS, which is complemented by the M/GLS test proposed by Ng-Perron (2001) is performed selected by VAR methodology of time series, are analyzed together which allowed to find evidence of the existence of a long-term relationship between the flows of foreign portfolio investment to Mexico and the EMBI index.
\end{abstract}

Keywords: Time Series Models, Cointegration, Unit Root, VAR, Financial Markets.

Clasificación JEL: C32, C320, E44

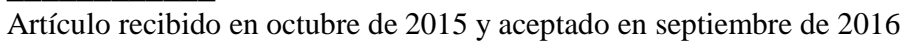

Artículo disponible en versión electrónica en la página www.revista-eea.net, ref. ə-35101 


\section{INTRODUCCIÓN}

Un tema relevante de investigación es sin duda la internacionalización de los mercados financieros en la esfera global, este trabajo se enfoca al análisis de los flujos de capital extranjero y su relación con la calificación del riesgo país en México. La inestabilidad económica, política y social son factores determinantes de la valuación del riesgo país, este es una suma de ponderaciones de una serie de los fundamentales macroeconómicos de un país en el corto plazo. Se encontró que en las dos últimas décadas los flujos de capital extranjero se han incrementado en forma considerable alcanzando los 831 mil millones de dólares, se analiza en conjunto la estabilidad macroeconómica asociada a la autonomía del Banco de México y las reformas jurídicas para la eliminación de controles fiscales a la entrada y salida de los capitales de corto plazo con el Reglamento de ley de Inversiones Extranjeras de 1989, estos factores se consideraron relevantes para establecer porque a la economía mexicana se le modificó la calificación de riesgo país por parte de las calificadoras internacionales entre ellas JP Morgan.

El interés por medir el riesgo país surge a principios de la década de los años 1980, cuando México en 1982 entra en una moratoria de pagos sobre la deuda externa soberana, como consecuencia de sufrir una crisis financiera por la caída de los precios internacionales del petróleo y el aumento de las tasas de interés internacionales, estos efectos se traducirían en una devaluación del peso y una severa crisis económico-financiera.

El proceso de globalización económica, el surgimiento de las economías emergentes, las vulnerabilidades económicas expuestas por los comportamientos negativos de los fundamentales económicos y la baja credibilidad en las políticas de estabilización hicieron necesaria una estratificación de los indicadores económicos por países para evitar en el corto plazo las crisis económicofinancieras. La génesis del riesgo país se establece en 1980 Polonia declaró la suspensión de pagos de su deuda soberana, después en México donde se presentaron cuatro crisis de ajuste del tipo de cambio 1982,1987,1988 y 1994, que ocasionó problemas financieros con los organismos internacionales y acreedores extranjeros generando problemas para acceder a nuevos créditos externos. En Asia-Pacifico 1997, la crisis financiera inició con un cambio de régimen cambiario en Corea del Sur y rápidamente se propagó hacia Malasia Singapur y Hong Kong, iniciando un ciclo de devaluación-inflación con correcciones de política monetaria y fiscal. En Rusia para 1998 su economía colapsó por una inflación alta originada por el ajuste cambiario respecto al dólar norteamericano. La crisis del real en Brasil para 1999 y del peso en Argentina en 2002 influenciaron en la región una gran pérdida de credibilidad en las políticas de estabilización económica y corrección de corto plazo, al generar una mayor incertidumbre financiera con los acreedores internacionales que exigieron más garantías para la compra de bonos soberanos de corto plazo. 
Bajo estas condiciones el análisis de riesgo país es utilizado por los bancos y corredurías de fondos de inversión internacionales para otorgar créditos en un país o en otros, la estratificación y ponderación de factores económicos, sociales y políticos surge para dar una mayor certidumbre y viabilidad en la compra de bonos de deuda soberana e instrumentos financieros comercializados en los mercados internacionales de capital.

México inicia un proceso de reestructuración económica de largo plazo a mediados de la década de los 80 , una de ellas fue la incursión en la esfera financiera internacional en la atracción de los flujos de capital extranjero para esto se modificó la Ley de Inversiones Extranjeras de 1973 en 1984 y se emitió el Reglamento de la Ley de Inversiones Extranjeras en 1989, con el objetivo de facilitar el ingreso de capital extranjero al sistema financiero. En la década de los años 90 se adoptó un régimen cambiario de libre flotación a partir de 1995 y una estrategia de tasas de interés internas acorde con los objetivos de la política monetaria para mantener un nivel de precios acorde con la política de estabilización macroeconómica en el corto y mediano plazo. En este periodo el interés por evaluar el riesgo país llevó a que la calificadora JP Morgan (1999) propusiera un índice para caracterizar el riesgo país de los bonos de los países emergentes. La calificación del riesgo país de JP Morgan (1999) se conoce como el Î́ndice del Mercado de Bonos de los Países Emergentes (Emerging Market Bond Index, EMBI) el cual genera una estratificación nominal e influye directamente en el flujo de Inversión Extranjera de Cartera Total (IECT) hacia la economía mexicana, aunque existen otros índices que miden el riesgo país, como ejemplos los índices Euromoney Country Risk ${ }^{1}$ (ECR), ICRG ${ }^{2}$ (International Country Risk Guide), Standard \& Poor Global Ratings, etc.

Como resultado de las políticas de estabilización se modificó la clasificación del riesgo país de México se refleja en los ratings del índice EMBI de JP Morgan y como consecuencia se incrementó el volumen de Inversión Extranjera de Cartera Total a 308, 222 millones de dólares en octubre de 2010 el flujo más alto en el periodo de estudio, el cual también está asociado a las crisis financieras de Argentina 2002, EUA 2008 y de la Eurozona 2010.

\footnotetext{
${ }^{1}$ Euromoney Country Risk es una publicación bianual, desde 1993, hace una evaluación política, económica y de riesgo estructural para 187 países, la calificación del crédito para los valores nominales se asignan a las calificaciones soberanas de Moody, Standard \& Poor y Fitch IBCA, esta puntuación se promedia y el promedio se pondera al 10\%, en www.euromoneycoubtryrisk. com

${ }^{2}$ La calificación International Country Risk Guide (ICRG) comprende 22 variables en tres subcategorías de riesgo: políticas, financieras y económicas. Se crea un índice separado para cada una de las subcategorías. El índice de riesgo político se basa en 100 puntos, el riesgo financiero en 50 puntos, y el riesgo económico en 50 puntos. Esta metodología es propuesta por The PRS Group, en www.prsgroup.com/us/methodologies/icrg.
} 
La Inversión Extranjera de Cartera Total (IECT) en México se integra de dos mercados, el primero es el mercado de renta fija donde se adquieren bonos de deuda y el segundo es el de renta variable en el cuál se adquiere capital social de las diferentes empresas que cotizan en la Bolsa Mexicana de Valores (BMV). El índice EMBI de JP Morgan (1999) se compone de variables políticas, sociales y económicas, de forma ponderada siendo el riesgo económico el de mayor peso, al establecer la calificación del riesgo país.

La propuesta de analizar las series de tiempo de la IECT y el índice EMBI mediante dos enfoques metodológicos que buscan establecer el orden de estacionariedad de los procesos en el tiempo, mediante el análisis de DickeyFuller GLS o ERS/GLS y los estadísticos de prueba M/GLS. Posterior al análisis de estacionariedad se aplica la metodología de Vectores Autorregresivos (VAR) con corrección de errores el cuál se denomina Vector Error Correction Model por sus siglas en inglés VECM, para el periodo de enero de 1998 a enero de 2012, se anexa al final de este documento la información estadística de ambas series de tiempo.

El documento se divide en cinco secciones, la primera presenta una introducción sobre el riesgo país financiero, la segunda sección se divide en dos, la primera trata los aspectos teóricos sobre los principales enfoques de la terminología del riesgo país y los flujos de capital; en la segunda sección se revisan los conceptos sobre las series de tiempo no estacionarias, raíces unitarias y rompimientos estructurales. En la tercera parte se dedica al desarrollo metodológico y la utilización de las técnicas econométricas empleadas. La cuarta sección se dedica al análisis de resultados, análisis gráfico y la dinámica de cointegración; por último se presentan las conclusiones de la investigación.

\section{REVISIÓN DE LITERATURA}

\subsection{El Riesgo País y los Flujos de Capital}

A mediados de la década de los años 60 surge el modelo Mundell-Fleming, que desarrolla una perspectiva teórica de la movilidad perfecta de capitales financieros entre las economías, cuando surge la posibilidad de sustituir bonos con diferentes tasas de interés y por consiguiente diferentes rendimientos, una leve variación de las tasas de rendimiento genera una entrada masiva de capitales al mercado con una mayor tasa de interés, con tipo de cambio fijo, por consiguiente implicaba ajustes en la balanza de pagos y las reservas internacionales, lo que genero para las economías las crisis del sector externo que se manifestaron en devaluaciones y ajustes de estabilización. A partir de principios de los años setenta es cuando los tipos de cambio se flexibilizan los ajustes en el sector externo presentó una mayor dinámica que implicó ajustes en el tipo de cambio, las tasas de interés y los precios. 
El interés por el riesgo país surge a principios de la década de los años 1980, a partir de que México ingresa en una moratoria de sus obligaciones financieras sobre la deuda soberana también Polonia con antelación entró en crisis económica por los préstamos conseguidos en el exterior, además surgieron problemas internos con los trabajadores y el régimen político; se declaró entonces la suspensión de pagos sobre su deuda soberana. En México se presentaron cuatro crisis de ajuste del tipo de cambio en 1982, 1987,1988 y 1994, que ocasionó problemas financieros con los organismos internacionales y acreedores extranjeros, la caída de los precios internacionales del petróleo principal fuente de ingresos públicos y el alza en las tasas de interés internacionales en las cuales estaba contratado los préstamos con el exterior. En Asia-Pacifico 1997, la crisis inició con un ajuste del régimen de tipo de cambio en Corea del Sur y rápidamente se propagó hacia Malasia Singapur y Hong Kong, el ciclo de devaluación-inflación con correcciones de política monetaria y fiscal, en Rusia 1998 la economía colapsó por una inflación alta originada por el ajuste cambiario respecto al dólar norteamericano. Las crisis en Brasil 1999 y Argentina 2002 influenciaron la credibilidad de las políticas de estabilización económica y corrección de corto plazo, al generar incertidumbre financiera con los acreedores internacionales que exigieron más garantías para la compra de bonos de corto plazo y pago de intereses moratorios.

Entonces la estratificación y ponderación de factores, económicos y políticos surge para darle certidumbre y viabilidad a la compra de bonos de deuda soberana y demás instrumentos financieros comercializados en los mercados internacionales de capital. La evaluación del riesgo país se reduce a una suma de ponderaciones subjetivas de variables para un periodo de tiempo determinado. Algunas otras clasificaciones combinan tres índices de riesgo país internacional para emitir sus propias valuaciones de los mercados emergentes.

García et al. (2000) definen la calificación de riesgo país como una medida de largo plazo para determinar la capacidad de una economía para atender regularmente y en los tiempos previamente establecidos, el pago de las obligaciones derivadas de su deuda soberana en moneda extranjera. Las dimensiones del riesgo país que se engloban en dos componentes determinantes primero el riesgo económico (capacidad de pago) y segundo el riesgo político (voluntad de cumplir con dichas obligaciones).

Ayala et al. (2002) definen al concepto de riesgo país como el que se asume por el hecho de suscribir o adquirir títulos de renta fija o variable, emitidos por entidades de un país extranjero, o por conceder préstamos o créditos a residentes de dicho país. Schuster (2005) determina que el riesgo país tiene implicaciones que muestran el nivel de riesgo de compra de bonos en un país en un momento determinado, la probabilidad de que al país le sea imposible responder a sus compromisos de pago de deuda, en capital e intereses, en los términos acordados. 
El análisis de la capacidad de pago de un país está basado en los factores económicos y políticos que afectan la probabilidad de pago.

Díaz et al. (2007) explican que un índice de riesgo país, mide la posibilidad de que un deudor extranjero sea incapaz de cumplir sus obligaciones financieras por motivos políticos o económicos, esto es consecuencia de variables políticas, económicas y sociales que afectan su solvencia.

García et al. (2006) determinan que el riesgo país es afectado por una tasa de interés real negativa, deprime el sistema financiero, desincentiva el ahorro interno y fomenta la fuga de capitales. Además el tipo de cambio real es el instrumento de evaluación de las políticas macroeconómicas y es el indicador de competitividad del país.

Mascareñas (2008) define al riesgo país como la referencia a la incertidumbre asociada al rendimiento de la inversión que surge al negociar con las empresas o instituciones de un Estado determinado. Es decir, trata de las posibles consecuencias negativas referentes al valor de los activos situados en dicho Estado o a los derechos de los residentes en el mismo, como consecuencia de alteraciones en las estructuras políticas, económicas y sociales del país en cuestión.

San-Martín-Albizuri et al. (2011) definen que la evaluación del riesgo país mediante un índice o calificación es la suma de ponderaciones subjetivas de los valores de una serie de variables para un periodo de referencia.

Existen diferentes formas de medir el riesgo país, uno es a través de índices sobre las tasas de interés, el tipo de cambio, la estabilidad macroeconómica y los componentes de credibilidad de la política económica elaborados por los bancos internacionales y las calificadoras de crédito. El índice más conocido es de JP Morgan (1999) el cuál estima los puntos porcentuales que alcanzan los países dentro de la composición del índice EMBI como referencia para seguir la evolución en el mercado de deuda soberana de los países emergentes, los rendimientos que son el diferencial en puntos base con respecto a una canasta de bonos libres de riesgo como son los Treasury Bills. Además de ponderar la liquidez de los instrumentos de deuda en dólares americanos, creando así una escala crediticia sobre los mercados emergentes con una combinación del ingreso per cápita y la reestructuración histórica de su deuda soberana.

En Schuster (2005) plantea que el índice más importante en el mercado para los derivados de crédito son los swap de incumplimiento de crédito (Credit Default Swap, CDS). Los swaps de incumplimiento de crédito sobre los bonos soberanos de largo plazo mexicanos (United Mexican States, UMS ${ }^{3}$ ) los cuales

3 El Gobierno Federal de México convocó el 17 de abril de 1996 a los tenedores de Bonos Brady denominados en dólares de los Estados Unidos de América (Bonos Par y Descuento), a una oferta de Intercambio de dichos Instrumentos por un nuevo Bono, a un plazo de treinta años, con 
cubren a los inversionistas ante un incumplimiento en el pago de la deuda por parte de México. Estos se han intercambiado en el mercado financiero desde el año 2000 por los bonos de restructuración de deuda del Plan Brady de 1989, implicando que los nuevos determinantes del riesgo país son variables de mercado, el precio de los CDS son una medida del riesgo país si se toman los diferenciales entre los UMS y los T-Bill's (notas del tesoro norteamericano), el cuál es una referencia entre los bonos libres de riesgo y los bonos que pagan un premio al riesgo.

Algunas medidas indicativas para el riesgo país son las propuestas de un premio al riesgo que sería la sobretasa que se paga por encima de la tasa libre de riesgo, la calificación riesgo país, en este caso sería el margen de la tasa de rendimiento de los bonos del tesoro estadounidense a 5, 10 y 30 años propuesto por Campbell et al. (1997). Para Campbell (1994) la varianza de la tasa de interés sobre el tiempo es una medida indicativa del riesgo país, Rosas (2000) y DíazBautista et al. (2003) utilizaron un spread de tasas de interés de bonos soberanos entre México y Estados Unidos para estimar la tasa de premio al riesgo menos la calificación riesgo país, con observaciones mensuales. Márquez et al. (2003) presentan una medida de riesgo-rentabilidad y un diferencial de tasas trimestrales como indicador del riego país para la IECT, Góchez (2005) analiza el flujo de inversión extranjera hacia México sin la variable de riesgo país, Díaz et al. (2009) utiliza un diferencial de tasas de interés como calificación del riesgo país en su análisis sobre la inversión extranjera en México, Schwert (2011) define la calificación riesgo país como una medida del cambio porcentual en las tasas de rendimiento.

\subsection{Series de Tiempo, Raíces Unitarias y Rompimientos Estructurales}

Se define un cambio estructural en una serie de tiempo cuando hay modificaciones instantáneas o permanentes, invariables e inesperadas en uno o más componentes estructurales debido a eventos específicos. Estos impactos aleatorios determinan el procedimiento para modelar la serie de tiempo antes y después de dicho evento, la presencia o ausencia auxilia a identificar algunas características del proceso generador de información. En ausencia de raíz unitaria (proceso estacionario) la serie de tiempo fluctúa alrededor de una media constate en el largo plazo, e implica que este proceso tiene una varianza finita la cual no depende del tiempo. La no estacionariedad en la generación de información, no tiene una propensión para regresar en el largo plazo a la tendencia determinista y la varianza de la serie depende del tiempo.

vencimiento en el año 2026. La primera oferta de UMS fue a 5 años a una tasa anual del $6 \%$. Departamento de Análisis de estrategias de mercado BBVA-Bancomer, Septiembre de 2002. 
La no estacionariedad en un proceso generador de datos tienen efectos permanentes por los shocks aleatorios que experimentan y estas siguen un comportamiento aleatorio. Un cambio estructural se caracteriza por perturbar de forma permanente el componente determinista de la serie de tiempo, lo cual provoca pérdidas de poder en la identificación del modelo y las pruebas, por eso una omisión del cambio estructural en una inspección gráfica del proceso en el tiempo puede conducir a una incorrecta especificación del modelo y no explicar adecuadamente el comportamiento de la secuencia.

El diseño de pruebas estadísticas para comprobar estacionariedad mediante la prueba de raíz unitaria y cambios estructurales de Perron (1989) se basa en los estudios de Nelson y Plosner (1982), en Perron (1989) presenta la metodología para el contraste estadístico de no estacionariedad de una serie de tiempo contra un cambio estructural, después Stock (1990) desarrolla la metodología para las pruebas tipo $\mathrm{M}$ sobre estimadores modificados de estadísticos para raíces unitarias y cambios estructurales, Bannerje, et al. (1992) retoman este planteamiento y proponen una prueba que permiten identificar un solo rompimiento estructural contra la alternativa de raíz unitaria. Perron y Vogelsang (1992) presentan un esquema de atípico aditivo de efecto instantáneo donde se examina si la serie posee raíz unitaria o es estacionaria y de atípico innovativo con efecto gradual que modifica la función de tendencia, Zivot y Andrews (1992) provén evidencia empírica de que las innovaciones temporales afectan el comportamiento de la serie de tiempo al suponer un punto de cambio estructural endógeno contra la hipótesis nula de raíz unitaria.

Lumsdaine y Papell (1997), Lee y Strazicich (1999) extienden la metodología para series de tiempo con cambio estructural al permitir dos rompimientos estructurales en presencia de raíz unitaria. Bai y Perron $(1998,2003)$ estiman y hacen pruebas de linealidad sobre modelos con múltiples cambios estructurales, Kapetanios (2002) diseña una prueba de raíz unitaria contra la alternativa dem rompimientos estructurales donde estima los valores críticos para el contraste estadístico incorporando la suma mínima de los residuales, posteriormente Ng y Perron $(1996,2001)$ proponen una prueba para un cambio en la función de tendencia y otra para un cambio de pendiente e intercepto, este es el principio de los estadísticos de tipo GLS Modificado (M/GLS) los cuales se establecen a partir de la prueba Phillps-Perron (1988) del estadístico $Z$. Perron y Rodríguez (2003, 2012) se basan en el contraste de raíz unitaria de Elliott et al. (1996) ERS/GLS y presentan una extensión de la prueba de raíz unitaria de DickeyFuller (1979), con este análisis estadístico de tipo M/GLS se busca mejorar la identificación de los componentes determinísticos, las raíces unitarias y el cambio estructural mediante una cuasidiferenciación sobre la serie de tiempo, donde se estima el parámetro de contraste estadístico al determinar un punto óptimo factible definido como MP]T, en este proceso se asume que los 
residuales de la estimación de la serie de tiempo se distribuyen idéntica e independientemente con media cero y varianza contaste, $e_{t} i i d \sim(0,1)$.

\section{METODOLOGÍA}

Las fluctuaciones macroeconómicas se han caracterizado a lo largo de las últimas décadas como cambios transitorios y permanentes que se caracterizan como choques estructurales, que se utilizan en el análisis de series de tiempo, la estacionariedad de una variable se presenta cuando la media y la varianza son constates a través del tiempo en el mismo proceso generador de datos (PGD), esto contra la hipótesis de raíz unitaria la cual establece que la varianza es creciente en el tiempo, al ampliarse las metodologías se encontró evidencia de que los choques estructurales modifican en el corto y largo plazo la evolución de la series macroeconómicas. El análisis de series de tiempo mediante la metodología VAR permite capturar la dinámica de la información, los ajustes de corto plazo y la relación de equilibrio en el largo plazo.

El planteamiento metodológico inicia con la identificación de las variables de estudio, que son: la inversión extranjera de cartera total en México la cual se integra por el mercado de renta fija o de corto plazo donde se negocian bonos de deuda y el mercado de renta variable o de largo plazo, en este último se puede adquirir capital social de las empresas que cotizan en la Bolsa Mexicana de Valores, la variable que caracteriza el riesgo país es el índice EMBI propuesto por JP Morgan (1999) que incorpora los riesgos políticos, sociales y económicos de forma ponderada, donde el riesgo económico recibe la mayor ponderación al establecer la calificación del riesgo país.

Con la ayuda del software Econometrics Views 8 (EViews 8) como segunda fase se procesan las series de tiempo de la IECT y el índice EMBI la linealización se realiza mediante la aplicación de logaritmo natural; el tercer paso corresponde a la aplicación de las pruebas econométricas para establecer si existe estacionariedad de las variables mediante los enfoques de Elliott et al. (1996) ERS/GLS y M/GLS de Ng-Perron (2001), la cuarta etapa consiste en la diferenciación de ambas series y la aplicación de la metodología VAR en primera diferencia, esto establece un modelo vectorial autorregresivo de corrección de errores y posteriormente se realiza el análisis sobre los resultados econométricos del modelo.

El esquema econométrico de la prueba de raíz unitaria de Elliott et al. (1996) ERS/GLS con tendencia e intercepto a nivel, la cual posee una mayor potencia estadística y se basada en el siguiente modelo:

$$
\Delta X_{t}^{d}=\gamma X_{t-1}^{d}+\sum_{j=1}^{k} \delta_{j} \Delta X_{t-j}^{d}+\varepsilon_{t}
$$


Cuando se impone un parámetro de no centralidad para $\bar{\alpha}=1+\bar{c} / T$, $\bar{c}=-13.5$, una serie de tiempo con tendencia, donde $X_{t}^{d}=X_{t}-\hat{\beta}_{0}-\hat{\beta}_{1} t$, $\left(\hat{\beta}_{0}, \hat{\beta}_{1}\right)$ se estiman mediante la regresión de $\bar{X}$ sobre $\bar{W}$, donde $W_{t}=(1, t)^{\prime}$ siendo:

$$
\begin{aligned}
& \bar{X}=\left[X_{1},(1-\bar{\alpha} L) X_{2}, \ldots,(1-\bar{\alpha} L) X_{T}\right] \\
& \bar{W}=\left[W_{1},(1-\bar{\alpha} L) W_{2}, \ldots,(1-\bar{\alpha} L) W_{T}\right]
\end{aligned}
$$

También el criterio de Ng-Perron (2001) M/GLS si se encuentra que las series son no estacionarias, se procede a corregirlas por medio del operador diferencias. La cuarta etapa definen los siguientes estadísticos tipo $\mathrm{M}$, desarrollados por Perron y Ng (1996) M/GLS donde se exploran las características de una serie de tiempo la cual puede converger a distintas tasas de normalización, estos parámetros se definen por:

$$
\begin{gathered}
M Z_{\alpha}^{G L S}=\left(T^{-1} \tilde{y}_{T}^{2}-s^{2}\right)\left(2 T^{-2} \sum_{t=1}^{T} \tilde{y}_{t-1}^{2}\right)^{-1} \\
M S B^{G L S}=\left(T^{-2} \sum_{t=1}^{T} \tilde{y}_{t-1}^{2} / s^{2}\right)^{1 / 2} \\
M Z_{t}^{G L S}=\left(T^{-1} \tilde{y}_{T}^{2}-s^{2}\right)\left(4 s^{2} T^{-2} \sum_{t=1}^{T} \tilde{y}_{t-1}^{2}\right)^{-1 / 2}
\end{gathered}
$$

Donde $\tilde{y}_{t}=y_{t}-\widehat{\psi}^{\prime} z_{t}$, el parámetro $\widehat{\psi}^{\prime}$ minimiza (8).

$$
\begin{gathered}
y_{t}=d_{t}+\mu_{t} \text {, para } t=0, \ldots, T, \\
\mu_{t}=\alpha \mu_{t-1}+v_{t}
\end{gathered}
$$

En el cuál $\left\{v_{t}\right\}$ es un proceso estacionario no observado con media cero. En (6), $d_{t}=\psi^{\prime} z_{t}$, donde $z_{t}$ es el conjunto de elementos determinísticos, para cualquier serie de tiempo $y_{t}$, con componente determinístico $z_{t}$, se definen los datos trasformados $y_{t}^{\bar{\alpha}}$ y $z_{t}^{\bar{\alpha}}$ por:

$$
y_{t}^{\bar{\alpha}}=\left(y_{0},(1-\bar{\alpha} L) y_{t}\right), z_{t}^{\bar{\alpha}}=\left(z_{0},(1-\bar{\alpha} L) z_{t}\right), t=0, \ldots, T,
$$

Donde se define al estimador $\hat{\psi}$ que minimiza:

$$
S^{*}(\psi, \bar{\alpha}, \delta)=\sum_{t=0}^{T}\left(y_{t}^{\bar{\alpha}}-\psi z_{t}^{\bar{\alpha}}\right)^{2}
$$


El valor minimizado se expresa por: $S(\bar{\alpha}, \delta)$. En el que $\delta$ es el parámetro del rompimiento estructural, siendo $T_{B}$ el punto de quiebre, $T_{B}=T_{\delta}$ para algún $\delta$ el cuál se encuentra en el intervalo $\{0,1\}$. El término $s^{2}$ es el estimador autorregresivo de densidad espectral en frecuencia cero de $v_{t}$ definido como:

$$
s^{2}=s_{e k}^{2} /(1-\hat{b}(1))^{2}
$$

En donde $s_{e k}^{2}=(T-k)^{-1} \sum_{t=k+1}^{T} \hat{e}_{t k}^{2}, \hat{b}(1)=\sum_{j=1}^{k} \hat{b}_{j}$, y $\hat{b}_{j}\left\{\hat{e}_{t k}\right\}$, se generan de la siguiente regresión:

$$
\Delta \tilde{y}_{t}=b_{0} \tilde{y}_{t-1}+\sum_{t=1}^{k} b_{j} \Delta \tilde{y}_{t-j}+e_{t k}
$$

El estadístico MPT, está definido primero por el parámetro de no centralidad el cuáles: $\bar{\alpha}=1+\bar{c} / T$, siendo $\bar{c}=-13.5$, para $y_{t}=\langle 1, t\rangle$,

$$
\operatorname{MPT}(c, \bar{c}, \delta)=\{S(\bar{\alpha}, \delta)-\bar{\alpha} S(1, \delta)\} / s^{2}
$$

Esta es la expresión del estadístico de punto óptimo factible para una distribución asintótica positiva.

El quinto paso, donde se estima el modelo de corrección de errores de un vector autorregresivo y se aplica la prueba de cointegración de Johansen (1996). El concepto de cointegración propuesto por Granger (1983) y ampliado por Engle y Granger (1987) comprueba la estacionariedad de los residuales de la estimación de un modelo de series de tiempo no estacionarias, esto permite establecer que las relaciones económicas de largo plazo tienden a un equilibrio en el largo plazo. El método más utilizado para el análisis de cointegración es el enfoque de máxima verosimilitud propuesto por Johansen $(1988,1991,1996)$ y Johansen y Juselius $(1990,1992)$. Este procedimiento se inicia a partir de un modelo vectorial autorregresivo para un conjunto de variables a nivel y estacionarias, el modelo VAR se puede reescribir sobre la forma funcional de un VECM con el enfoque de ajuste dinámico en el corto plazo y una relación de equilibrio en el largo plazo, mientras que las variables se expresen en primeras diferencias.

Los estudios realizados sobre los flujos de Inversión Extranjera de Cartera hacia México por Rosas (2000) y Díaz-Bautista et al. (2003) consideran un modelo AR (3) con información mensual, Márquez et al. (2003) emplean un VECM, Góchez (2005) desarrolla un modelo ARIMA sin la variable riesgo país, Díaz et al. (2009) un VECM por tramos, todos ellos con datos trimestrales, estos modelos de estimación analizan los flujos de capital hacia la economía mexicana y encuentran evidencia empírica. Se propone que la técnica econométrica de análisis para estimar la forma funcional entre el EMBI y la IECT 
sea un VECM con observaciones mensuales para el periodo de enero de 1998 hasta enero de 2012.

Se define el vector autorregresivo con corrección de errores, el cual se presenta en su forma estructural como sigue:

$$
\begin{aligned}
& \operatorname{IECT}_{t}=\alpha_{1}+\delta_{t-i}+\sum_{i=1}^{t} \beta \Delta E M B I_{t-i}+\mu_{1 t} \\
& E M B I_{t}=\alpha_{2}+\delta_{t-i}+\sum_{i=1}^{t} \rho \Delta I E C T_{t-i}+\mu_{2 t}
\end{aligned}
$$

Donde $\delta_{t-i}$ es el parámetro de corrección de errores al utilizar la técnica VAR, para el análisis de estas series de tiempo, si las series de Tiempo del Índice EMBI y la IECT no son estacionarias a nivel, entonces el modelo se convierte en un VECM.

El objetivo de esta investigación fue encontrar evidencia empírica de que la calificación riesgo país medida a través del índice EMBI de JP Morgan (1999)fue un factor determinante de la atracción de inversión extranjera de cartera al mercado financiero mexicano en el periodo de enero 1998 a enero de 2012.

\section{ANÁLISIS DE RESULTADOS}

La Figura 1, presenta la evolución de la Inversión Extrajera de Cartera Total (IECT) en México para el periodo de enero de 1998 hasta enero de 2012 y la calificación riesgo país alcanzada en el Î́ndice EMBI de JP Morgan, se pueden observar las variaciones de ambas variables con una fuerte relación inversa, cuando el Índice EMBI disminuye implica que la IECT aumenta, esto explica que el coeficiente de correlación de Pearson alcance el -0.746885.

Los choques exógenos que experimenta la economía mexicana a través de las crisis desarrolladas en otras economías emergentes implican un aumento de la calificación del riesgo país para México, la crisis financiera de Asia-Pacifico en 1998 lleva el riesgo país a los 1,377 puntos en el EMBI, Brasil 1999-2000 y Argentina 2002 sitúan este indicador de riesgo soberano en los 995 y 938 puntos en la escala EMBI. La crisis financiera en los EUA de 2008 llevo a los 689 puntos en el índice EMBI a la economía mexicana, pero la crisis en la Eurozona de 2010 impacto positivamente el flujo de capital y la IECT aumento hasta los 308 mdd, después está retorno a su tendencia de largo plazo. Además la disminución del flujo de capital hacia la economía mexicana contrasta con una calificación del riesgo país muy alta entre enero de 1998 hasta enero de 2005. El aumento de la IECT es creciente cuando la calificación del riesgo país tiende a la baja en el periodo de septiembre de 2004 hasta enero 2012. 
Figura 1

Inversión Extranjera de Cartera Total en México e Índice Global en los Mercados Emergentes de JP Morgan: 1998:01 a 2012: 01

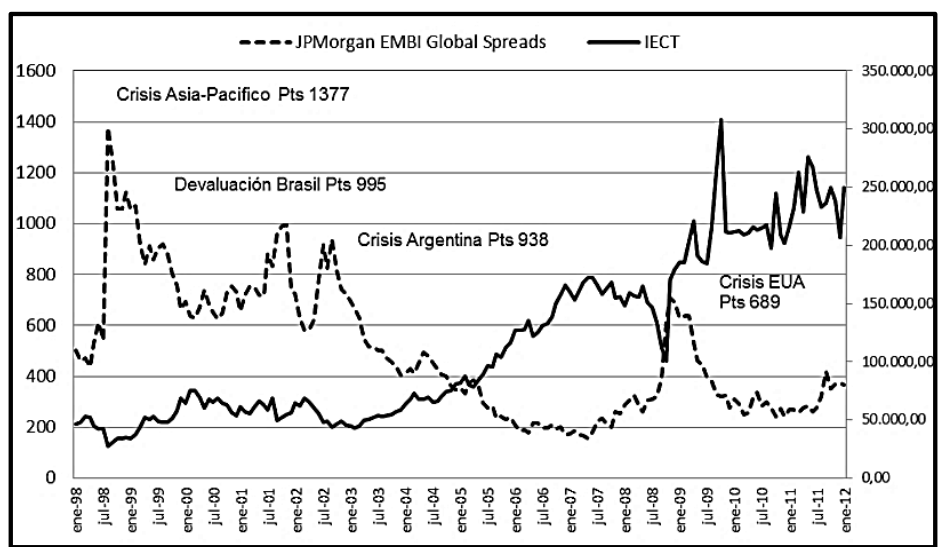

Fuente: elaboración propia con datos de JP Morgan y BMV.

En la Tabla 1 presentamos las estadísticas descriptivas de las series de tiempo empleadas en el modelo de corrección de errores, sin la aplicación de la transformación logarítmica, la mediana en ambas series es una medida robusta de centralidad de la distribución que es menos sensitiva a las innovaciones de la media. La IECT presenta un coeficiente de variación de más del 50 \% y en el caso del EMBI este parámetro alcanza más del 60 \% de variabilidad con respecto a su media.

Tabla 1

Estadísticas Descriptivas del EMBI y la IECT 1998:01-2012:01

\begin{tabular}{|l|r|r|}
\hline \multicolumn{1}{|c|}{ Estadístico } & \multicolumn{1}{c|}{ EMBI } & \multicolumn{1}{c|}{ IECT } \\
\hline Media & 498.662 & $115,234.6$ \\
\hline Máximo & 1377.000 & $308,222.1$ \\
\hline Mínimo & 155.000 & $27,655.24$ \\
\hline Desviación Estándar & 260.768 & $70,335.23$ \\
\hline Coeficiente de Variación & 0.52293 & 0.610355 \\
\hline Observaciones & 169 & 169 \\
\hline
\end{tabular}

Fuente: Elaboración propia con información de la BMV y JP Morgan.

La prueba ERS/GLS (1996) es la prueba de raíz unitaria de mayor potencia y busca establecer si existe la no correlación serial, normalidad y homocedasticidad de la serie de tiempo al analizar los errores. Las variables EMBI y la IECT se trasformaron mediante logaritmo natural para minimizar la varianza en estas. 
La elección del componente autorregresivo de orden $k$ se basa en la condición desarrollada por Ng-Perron (1996) donde se elige a $k$ como una función determinista de Tpor medio de la relación:

$$
k=\operatorname{int}\left\{c(T / 100)^{1 / d}\right\}
$$

Basándose en los valores obtenidos por Schwert (1989) de un análisis de simulaciones de Monte Carlo,se eligió ac $=4$ y $d=4$ que es una regla utilizada para pruebas de raíz unitaria en series de tiempo, bajo este criterio se seleccionac $=4$ y se obtuvieron los siguientes valores para el parámetro del número de rezagos $k$ :

Tabla 2

Número de Rezagos para las series de tiempo de la IECT y el Índice EMBI

\begin{tabular}{|c|c|c|}
\hline$C$ & $T$ & $k$ \\
\hline 4 & 169 & 4.560 \\
\hline
\end{tabular}

Fuente: Elaboración propia en base a la información de la BMV y JP Morgan.

La longitud del rezago es de $k=5$, lo que permite obtener los resultados para la prueba de raíz unitaria con intercepto y tendencia lineal de las dos series en estudio, que se presentan en la Tabla 2, los resultados implican que las series poseen raíz unitaria y son no estacionarias a nivel, las variables evolucionan alrededor de una tendencia determinística lineal. Los resultados confirman que no se puede rechazar la hipótesis nula donde las series de tiempo del EMBI y la IECT poseen raíz unitaria a un nivel de significancia del $0.05 \%$. Esta prueba se basa en una distribución asintótica negativa donde el valor del parámetro de contraste del EMBI es mayor que el estadístico $t$ reportado por Elliott et al. (1996) al $0.05 \%$, lo cual implica que el resultado cae en la zona de no rechazo de $H o$, así también no se puede rechazar la hipótesis nula para la IECT.

Tabla 3

Prueba de Raíz Unitaria ERS/GLS con intercepto y tendencia lineal, a Nivel

\begin{tabular}{|c|c|c|c|}
\hline Variable & Estadístico t & $\begin{array}{c}\text { Coeficiente de los } \\
\text { Residuales GLS }\end{array}$ & $\begin{array}{c}\text { Longitud del } \\
\text { rezago }\end{array}$ \\
\hline EMBI & -2.103850 & -0.052127 & 5 \\
\hline IECT & -2.046207 & -0.070599 & 5 \\
\hline $\begin{array}{l}\text { La hipótesis nula es, Ho: la serie pose una Raíz Unitaria. Valores críticos de prueba: al 1\% }-3.504400,5 \% \\
-2.96700 \text { y al 10\% -2.67700 }\end{array}$ \\
\hline \multicolumn{2}{l}{ Prueba de Raíz Unitaria ERS/GLS de Elliott, Rothenberg y Stock (1996) } \\
\hline
\end{tabular}

Fuente: Estimación propia con información de JP Morgan y la BMV.

Cuando se aplica la prueba ERS/GLS (1996) a la trasformación de las series de tiempo mediante la primera diferencia, se corrige la correlación serial y se 
normalizan los errores de las variables, los resultados que se presentan en la Tabla 3,implican que las series ya no poseen raíz unitaria, son estacionarias y evolucionan alrededor de una linealidad determinística en una distribución asintótica negativa, a un nivel de significancia del $0.05 \%$ que permite rechazar la hipótesis nula de que las series de tiempo posen raíz unitaria y por lo tanto son estacionarias en primera diferencia.

\section{Tabla 4}

Prueba de Raíz Unitaria ERS/GLS con intercepto $y$ tendencia lineal, en Primera diferencia

\begin{tabular}{|c|c|c|c|}
\hline Variable & Estadístico t & $\begin{array}{c}\text { Coeficiente de los } \\
\text { Residuales GLS }\end{array}$ & $\begin{array}{c}\text { Longitud del } \\
\text { rezago }\end{array}$ \\
\hline EMBI & -4.835823 & -0.792758 & 5 \\
\hline IECT & -5.633241 & -1.168860 & 5 \\
\hline $\begin{array}{l}\text { La hipótesis nula es, Ho: la serie pose una Raíz Unitaria. Valores críticos de prueba: al 1\% } \\
-2.96800 \text { y al 10\% -2.67800 }\end{array}$ \\
\hline \multicolumn{2}{|l}{ Prueba de Raíz Unitaria ERS/GLS de Elliott, Rothenberg y Stock (1996) } \\
\hline
\end{tabular}

Fuente: Estimación propia con información de JP Morgan y la BMV.

En la Tabla 4, se presentan los resultados del análisis de raíz unitaria de NgPerron (2001) que es un estadístico de tipo M/GLS, la prueba busca eliminar los componentes determinísticos, las raíces unitarias y el cambio estructural mediante una cuasidiferenciación, donde se estima con un punto óptimo factible MPT, este parámetro permite rechazar la existencia de correlación serial en los residuales y la eliminación de la tendencia en la serie de tiempo, los resultado se presentan en la cuarta columna y por consiguiente se rechaza la hipótesis de tendencia en el periodo de análisis para los datos del Índice EMBI y la IECT.

\section{Tabla 5}

Prueba de Raíz Unitaria M/GLS, en primera diferencia, con intercepto y tendencia lineal

\begin{tabular}{|c|c|c|c|c|}
\hline Variable & $M Z_{\alpha}$ & $M Z_{t}$ & $M S B$ & MPT \\
\hline EMBI & -83.1554 & -6.44592 & 0.07752 & 1.10474 \\
\hline IECT & -83.3114 & -6.41356 & 0.07698 & 1.26069 \\
\hline \multicolumn{5}{|c|}{$\begin{array}{l}\text { La Hipótesis Nula, Ho: la serie de tiempo posee raíz unitaria. Valores críticos de prueba de la Tabla 1, } \\
\text { Ng-Perron (2001) }\end{array}$} \\
\hline & $M Z_{\alpha}$ & $M Z_{t}$ & $M S B$ & MPT \\
\hline $1 \%$ & -23.8000 & -3.42000 & 0.14300 & 4.03000 \\
\hline $5 \%$ & -17.3000 & -2.91000 & 0.16800 & 5.48000 \\
\hline $10 \%$ & -14.2000 & -2.62000 & 0.18500 & 6.67000 \\
\hline \multicolumn{5}{|c|}{ Prueba de Raíz Unitaria M/GLS de Ng-Perron (2001) } \\
\hline
\end{tabular}

Fuente: Estimación propia con información de JP Morgan y la BMV.

El estadístico $M Z_{\alpha}$, corresponde al estimador de la prueba de autocorrelación serial en los residuales yse presentan en la primera columna de la Tabla 4, donde las variables EMBI e IECT están en la primera diferencia, estos resultados 
confirman que se rechaza a un nivel de significancia del $0.05 \%$ la autocorrelación serial en los residuales. La prueba $M Z_{t}$, se realiza sobre los errores de la estimación y evalúan si estos siguen un camino aleatorio en una regresión OLS contra la hipótesis alternativa de que los residuales son generados por un proceso de Markov estacionario de primer orden, los resultados de la columna 2 de la Tabla 5, muestran que para un nivel de confianza del $0.95 \%$ los residuales son estacionarios. El estadístico MSB de la tercera columna se relaciona de la siguiente forma con los parámetros $M Z_{\alpha}$ y $M Z_{t}$, con lo que se evalúa la no correlación serial y la estacionariedad de los residuales $M Z_{t} \approx M S B * M Z_{\alpha}$, (en el análisis se sustituyen los valores del EMBI y se obtiene $(-83.1554 * 0.07752) \approx-$ 6.44592, que es el primer parámetro de la columna 2 de la Tabla 5).

La ecuación 13.1 y 14.1 del vector de corrección de errores se expresan en forma funcional al sustituir los valores obtenidos en la regresión del VECM:

$$
\begin{gathered}
\Delta^{2} I E C T 1_{t}=0.0155+10.7919 * \Delta E B I 1_{t-1}+\delta \Delta I E C T 1_{t-1}+\sum_{i=1}^{6} \alpha_{i} \Delta^{2} I E C T 1_{t-1}+\sum_{i=1}^{6} \beta_{i} \Delta^{2} E M B I 1_{t-1} \\
\Delta^{2} E M B I 1_{t}=0.0155+10.7919 * \Delta E B I 1_{t-1}+\delta \Delta I E C T 1_{t-1}+\sum_{i=1}^{6} \rho_{i} \Delta^{2} I E C T 1_{t-1}+\sum_{i=1}^{6} \theta_{i} \Delta^{2} E M B I 1_{t-1}
\end{gathered}
$$

En la Tabla 6 se presentan los resultados de VECM de integración de orden uno entre la IECT y el índice EMBI, cinco choques exógenos se presentaron durante el periodo de estudio, las crisis financieras de Asia-Pacifico 1997-1998, Brasil y Rusia 1999, Argentina 2002, Estados Unidos 2008 y la Eurozona 2010, lo cual atrajo un mayor flujo de capital hacia la economía mexicana, como un mercado emergente con credibilidad y estabilidad macroeconómica a través de un equilibrio entre las tasas de interés y el ajuste de mercado en el tipo de cambio al pasar de un esquema de bandas de flotación al régimen de flotación libre a partir de la crisis del peso mexicano a finales de 1994, esto permitió que no existiera un sobrecalentamiento de la economía, por un mayor ingreso de capital extranjero de corto plazo y la calificación de riesgo país medida a través del Índice EMBI de JP Morgan (1999) está tendiera a disminuir desde septiembre de 1998 hasta mayo de 2009. Además los parámetros estimados para el VECM de la IECT y el Î́ndice EMBI son estadísticamente significativos, también se corrobora la relación inversa que existe entre el coeficiente de corrección o normalización de errores entre las variables IECT y EMBI en el periodo de estudio que comprende de enero de 1998 hasta enero de 2012.

Se realiza el análisis de cointegración de Johansen (1996), los resultados de esta prueba se presenta en la Tabla 7, donde encontramos al menos dos vectores de cointegración, a un nivel del $0.05 \%$ se puede rechazar la hipótesis de no cointegración entre la Inversión Extranjera de Cartera Total en México y el Índice EMBI de JP Morgan. 
Tabla 6

Vector de Cointegración para la IECT 1998:01-2012:01

\begin{tabular}{|c|c|c|c|c|c|c|c|}
\hline & IECT & EMBI & & $\begin{array}{l}\text { Coeficientes de } \\
\text { Corrección }\end{array}$ & IECT & EMBI & \\
\hline Coeficiente & 1.000 & 10.7495 & & & 0.0506 & -0.1079 & \\
\hline Error Estándar & & 1.5507 & & & 0.1594 & 0.0161 & \\
\hline Estadístico t & & 6.9272 & & & 3.1793 & -6.6706 & \\
\hline$\Delta^{2} I E C T 1_{t}$ & $\begin{array}{l}\text { Término de } \\
\text { CE }\end{array}$ & $\Delta^{2} I E C T 1_{t-1}$ & $\Delta^{2} I E C T 1_{t-2}$ & $\Delta^{2} I E C T 1_{t-3}$ & $\Delta^{2} I E C T 1_{t-4}$ & $\Delta^{2} I E C T 1_{t-5}$ & $\Delta^{2} I E C T 1_{t-6}$ \\
\hline Coeficiente & 0.05068 & -0.90026 & -0.73842 & -0.70723 & -0.51319 & -0.37011 & -0.23428 \\
\hline Error Estándar & -0.01594 & -0.08909 & -0.11349 & -0.12601 & -0.12647 & -0.11255 & -0.08723 \\
\hline \multirow[t]{2}{*}{ Estadístico t } & 3.17936 & -10.1049 & -6.50635 & -5.61231 & -4.05782 & -3.28851 & -2.68579 \\
\hline & & $\Delta^{2} E M B I 1_{t-1}$ & $\Delta^{2} E M B I 1_{t-2}$ & $\Delta^{2} E M B I 1_{t-3}$ & $\Delta^{2} E M B I 1_{t-4}$ & $\Delta^{2} E M B I 1_{t-5}$ & $\Delta^{2} E M B I 1_{t-6}$ \\
\hline Coeficiente & & -0.382047 & -0.267083 & -0.347285 & -0.194205 & -0.132883 & -0.037379 \\
\hline Error Estándar & & -0.16188 & -0.1515 & -0.14033 & -0.12812 & -0.10528 & -0.07747 \\
\hline Estadístico t & & -2.36001 & -1.76295 & -2.47479 & -1.51582 & -1.26223 & -0.48247 \\
\hline$\Delta^{2} E M B I 1_{t}$ & & $\Delta^{2} I E C T 1_{t-1}$ & $\Delta^{2} I E C T 1_{t-2}$ & $\Delta^{2} I E C T 1_{t-3}$ & $\Delta^{2} I E C T 1_{t-4}$ & $\Delta^{2} I E C T 1_{t-5}$ & $\Delta^{2} I E C T 1_{t-6}$ \\
\hline Coeficiente & -0.10793 & 0.09842 & -0.06297 & 0.14152 & 0.20919 & 0.13768 & 0.05851 \\
\hline Error Estándar & -0.01618 & -0.09043 & -0.1152 & -0.12791 & -0.12837 & -0.11424 & -0.08854 \\
\hline \multirow[t]{2}{*}{ Estadístico t } & -6.67065 & 1.08842 & -0.54663 & 1.10644 & 1.62959 & 1.20516 & 0.66088 \\
\hline & & $\Delta^{2} E M B I 1_{t-1}$ & $\Delta^{2} E M B I 1_{t-2}$ & $\Delta^{2} E M B I 1_{t-3}$ & $\Delta^{2} E M B I 1_{t-4}$ & $\Delta^{2} E M B I 1_{t-5}$ & $\Delta^{2} E M B I 1_{t-6}$ \\
\hline Coeficiente & & 0.19744 & 0.10464 & 0.23181 & 0.25773 & 0.16505 & 0.07655 \\
\hline Error Estandar & & -0.16432 & -0.15378 & -0.14244 & -0.13005 & -0.10686 & -0.07864 \\
\hline \multirow[t]{2}{*}{ Estadístico t } & & 1.20159 & 0.68047 & 1.62742 & 1.98182 & 1.54454 & 0.97348 \\
\hline & IECT & EMBI & & IECT & EMBI & & \\
\hline$R^{2}$ & 0.54052 & 0.61245 & $\bar{R}^{2}$ & 0.49989 & 0.57818 & & \\
\hline
\end{tabular}

Fuente: Estimación propia con información de la BMV y JP Morgan.

Los valores críticos de prueba del estadístico de la Traza son muy superiores a los valores de prueba presentados por MacKinnon et al. (1999) para rechazar la hipótesis nula de no cointegración entre la IECT en México y el Índice EMBI de JP Morgan de la calificación riesgo país.

\section{Tabla 7}

Muestra (ajustada): 1998M09 2012M01

Supuesto de Tendencia: Tendencia lineal determinística

Series: D(EMBI1) D(IECT1)

Intervalo del rezago (en primeras diferencias): 1 a 2, 3 a 4, 5 a 6

Prueba de Rango de Cointegración no restringido (Traza)

\begin{tabular}{|c|c|c|c|c|}
\hline Ho & Eigenvalue & $\begin{array}{c}\text { Traza } \\
\text { Estadístico } \lambda\end{array}$ & Valor Critico al $\mathbf{0 . 0 5} \%$ & Probabilidad** $^{*}$ \\
\hline Ninguna * & 0.234862 & 78.60803 & 15.49471 & 0.0000 \\
\hline Al menos 1 * & 0.197922 & 35.50849 & 3.841466 & 0.0000 \\
\hline $\begin{array}{l}\text { * Denota el rechazo de la Hipótesis nula (Ho)al } \\
\text { cointegración al 0.05\%. La prueba estadística indica que existen 2 vectores de } \\
\text { MacKinnon-Haug-Michelis (1999). }\end{array}$
\end{tabular}

Fuente: Estimación propia con información de JP Morgan y la BMV. 
Los resultados del VECM que se presentan en la Tabla 7, una primera afirmación sobre la relación estructural entre el índice EMBI y la IECT en México, se encuentra en el espacio generado por los dos vectores de cointegración donde se muestra que existe una relación inversa entre estas dos variables, lo que implica que un mayor riesgo país genera una mayor exposición al riesgo y tiene un sentido económico muy fuerte ya que los capitales de corto plazo en la esfera global buscan una mayor tasa de rendimiento a sus inversiones.

En la Figura 2, muestra la descomposición de la varianza que provee información acerca de la importancia relativa de cada innovación aleatoria o choque aleatorio endógeno o exógeno que afectan a las variables de un sistema de vectores autorregresivos, la descomposición de Cholesky de impulsorespuesta ante innovaciones (impactos aleatorios) en las series de tiempo son fundamentales en esta metodología, se observa que un cambio o innovación aleatoria (aumento de la calificación del riesgo país) de una desviación estándar del EMBI implica que el ajuste sea de tan solo cuatro periodos (meses) para que el flujo de IECT se estabilice nuevamente como se muestra de forma gráfica

Figura 2

Respuesta de la IECT ante una innovación del Índice EMBI de JP Morgan

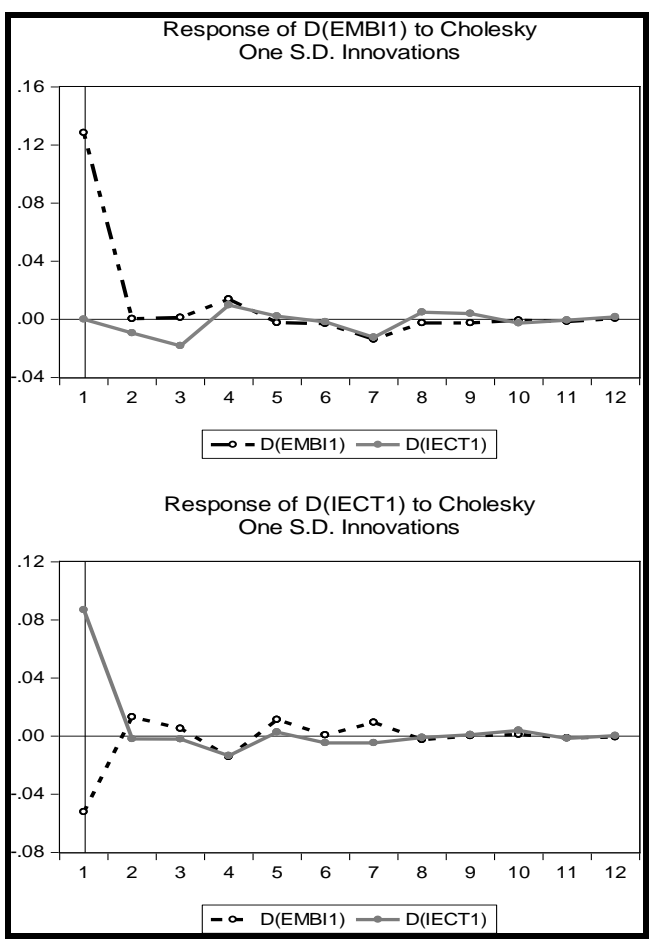

Fuente: Estimación propia con información de JP Morgan y la BMV. 
Esta descomposición de la varianza se basa en la inversa de la matriz de varianzas-covarianzas de ortogonalización de los impulsos o innovaciones aleatorios que puede experimentar una serie de tiempo con el factor de Cholesky en el análisis de los sistemas VAR.

En la Figura 2, se observa que un cambio en la IECT implica un ajuste de igual tiempo en el EMBI para la estabilización en el corto plazo del flujo de capital, sin pérdida de generalidad el índice tarda en asimilar el ajuste en el mismo periodo de tiempo, que si se produjera una innovación en el EMBI que afecta directamente al flujo de capital hacia la economía mexicana en una mayor proporción, debido a que las expectativas y el ajuste dinámico en el corto plazo repercute sobre todo por el entorno económico y financiero con una creciente incertidumbre y una mayor calificación en el riesgo país a través del índice EMBI .

\section{CONCLUSIONES}

A partir del estudio del comportamiento del índice EMBI se encontró evidencia empírica de su relación con la inversión extranjera de cartera en México, la técnica de análisis de causalidad de series de tiempo se aplicó al periodo de estudio, se identificó la presencia de eventos exógenos que modificaron la dinámica de los flujos de capital en los países emergentes en particular en México que se presenta un cambio de tendencia abrupto en febrero de 2002. Los resultados del estudio permiten afirmar lo siguiente:

La política económica de México busco desde hace más de dos décadas promover un proceso de competencia con otros mercados emergentes para captar los flujos de capital extranjero para darle certidumbre al crecimiento y financiación de la estructura económica del país en el largo plazo, el análisis denota que el flujo de capitales ha crecido con una tendencia de $1.6 \%$ mensual que no se ha reflejado en el crecimiento económico y el financiamiento de actividades productivas, lo que indica una clara concentración en el mercado de renta variable.

La reforma jurídica referente a la Inversión Extranjera de 1984 y el Reglamento de la Ley sobre Inversiones Extranjeras publicado en 1989, se complementó con el régimen cambiario de libre flotación desde 1995 y la estrategia de tasas de interés internas acorde con los objetivos de la política monetaria de mantener un nivel de precios para la estabilización macroeconómica después de la crisis del peso de 1994, se reflejó en el aumento de los flujos externos de capital y la reducción del riesgo país como lo denota una mejor posición en la calificación del Índice EMBI, la dinámica del índice refleja un valor máximo en septiembre de 1998 como consecuencia de crisis financiera de Asia Pacifico, a partir de mayo de 2005 se observa una reducción constante en sentido inverso al flujo de inversión extranjera de cartera, en septiembre de 2008 
el riesgo país crece a consecuencia de la crisis financiera de EUA que afecto de forma transitoria el flujo de capital extranjero, la rápida recuperación de los flujos está ligada a la estabilidad de las variables macroeconómicas nominales.

Los resultados del estudio se obtuvieron mediante las prueba de hipótesis para identificar raíces unitarias, rompimientos estructurales y tendencia temporal en las series de tiempo de la IECT y el Índice EMBI. Se afirma a partir de las pruebas que las series no son estacionarias a nivel y por lo tanto evolucionan alrededor de una tendencia determinística lineal en el periodo de enero 1998 a enero de 2012, adicionalmente es posible eliminar las observaciones atípicas de las series de tiempo que se identifican por innovaciones en la estructura de las variables que se refleja por la presencia de autocorrelación serial en los residuales de la regresión. Al aplicar las pruebas ERS/GLS (1996) y M/GLS (2001), se rechazó la no estacionariedad de las series tiempo en primera diferencia.

Las series del EMBI y la IECT presentan dos vectores de cointegración que permiten establecer una relación estructural entre ambas variables la cuáles inversa y se determina fundamentalmente por el riesgo país de México y la rentabilidad del mercado financiero nacional. La robustez estadística al modelo econométrico de corrección de errores se confirma con el estadístico tque se presentan en la Tabla 5. El ajuste en el corto plazo que tendría la IECT ante una variación exógena de la calificación riesgo país, implica un ajuste dinámico de tan solo cuatro meses, esta es una interpretación económica del proceso de ajuste en el corto plazo, entre el riesgo país y el costo del dinero representado por la tasas de interés.

La evidencia empírica presenta que la cointegración de orden I (1) y los choques estructurales o innovaciones aleatorias representan una relación de equilibrio en el largo plazo entre el índice EMBI y la IECT hacia la economía mexicana presenta un ajuste dinámico de cuatro periodos con el índice EMBI.

\section{REFERENCIAS BIBLIOGRÁFICAS}

AYALA CALVO, JUAN CARLOS, ITURRALDE JAINAGA, TXOMIN y RODRÍGUEZ CASTELLANOS, ARTURO (2002). "Construcción de índices simplificados de riesgo país: Aproximación a los casos de Europa y América". Cuadernos de Gestión Vol. 2. Núm. 2, pp. 79-100.

BAI, JUSHAN and PERRON, PIERRE (1998). "Estimating and Testing Linear Models with Multiple Structural Changes". Econometrica, Vol. 66, No. 1, pp. 47-78.

BAI, JUSHAN and PERRON, PIERRE (2003). "Critical values for multiple structural changes". Econometrics Journal, Vol. 6, pp. 72-78.

BANERJEE, ANINDYA, LUMSDAINE, ROBIN L. and STOCK, JAMES H. (1992). "Recursive and Sequential Tests of the Unit-Root and Trend-Break Hypotheses: 
Theory and International Evidence". Journal of Business \& Economic Statistics, Vol. 10, No. 3, pp. 271-287.

BHARGAVA, A (1986). "On the Theory of Testing for Unit Roots in Observed Time Series". Review of Economic Studies, 53, pp. 369-384.

CAMPBELL, R. HARVEY (1994). "Predictable risk and returns in emerging markets". NBER, num. 4621, p. 57.

CAMPBELL, JOHN y., LO, ANDREW W. and MACKINLAY, A. CRAIG (1997). The Econometrics of Financial Markets. Princeton University Press, pp. 135-425.

DÍAZ-BAUTISTA, ALEJANDRO y ROSAS CHIMAL, MARIO ALBERTO (2003). "Los Determinantes de la Inversión Extranjera de Cartera en México, un Análisis de Corte Estructural". Comercio Exterior, Vol. 53, núm. 3 pp. 214-228.

DÍAZ GONZALEZ, ELIZEO y RUVALCABA, ANGELITA (2009). "La Inversión Extranjera de Cartera en el Marco de la Liberalización Financiera en México". Comercio Exterior, México, pp. 534-550.

DÍAZ TAGLE, SEBASTIÁN, GALLEGO CHECA, ALICIA y PALLICERA SALA, NEUS (2007). "Riesgo País en Mercados Emergentes". IDEC Universidad Pompeu Fabra, p.43.

DICKEY, D.A. and FULLER, W.A (1979). "Distribution of the Estimators for Autoregressive Time Series with a Unit Root". Journal of the American Statistical Association, num. 74, pp. 427-431.

ELLIOTT, G., ROTHENBERG, T. J. and STOCK, J. H (1996). "Efficient Tests for an Autoregressive Unit Root". Econometrica 64, pp. 813-836.

ENGLE, R. and GRANGER, C. (1987). "Cointegration and error correction: representation, estimation, and testing". Econometrica 55, pp. 251-276.

GARCÍA GÁMEZ, SOFÍA y VICÉNS OTERO, JOSÉ(2000). "Especificación y Estimación de un Modelo de Riesgo País". Departamento de Economía Aplicada, Universidad Autónoma de Madrid, p. 23.

GARCÍA GÁMEZ, SOFÍA y VICÉNS OTERO, JOSÉ (2006). "Factores Condicionantes en la Medición del riesgo Soberano en los Países Emergentes". Estudio de Economía Aplicada, Vol. 24(I), pp. 245-272.

GÓCHEZ SEVILLA, ROBERTO ABRAHAM (2005). Inversión Extranjera de Cartera: Determinantes en México. UAM-I, México 01/2005. p.95.

GRANGER, C. W. J (1969). "Investigating Causal Relation by econometrics models and Cross-Spectral Methods". Econometrica, num. 37, pp. 424-438.

GRANGER, C. W. J (1983). Forecasting white noise. In Zellner, A. (edt.). Applied Time Series Analysis of Economic Data. pp. 308-314.

INDICADORES BURSÁTILES DE LA BOLSA MEXICANA DE VALORES. Enero 1989 a Diciembre 2011, publicación mensual.

JOHANSEN, S. (1988). "Statistical analysis of cointegration vectors". Journal of Economic Dynamics and Control ,12, pp. 231-254.

JOHANSEN, S. (1991). "Estimation and hypothesis testing of cointegration vectors in Gaussian vector autoregressive models". Econometrica, 59, pp. 1551-1580.

JOHANSEN, S. (1996). Likelihood-based Inference in Cointegrated Vector Autoregressive Models. Oxford University Press, New York. 
JOHANSEN, S. and JUSELIUS, K. (1990). "Maximum likelihood estimation and inference on cointegration With application to the demand for money". Oxford Bulletin of Economics and Statistic, 52, pp.169-210

JOHANSEN, S. and JUSELIUS, K (1992). "Testing structural hypotheses in a multivariate cointegration analysis of the PPP and the UIP for UK". Journal of Econometrics 53, pp. 211-244.

JP MORGAN (1999). "Introducing the JP Morgan Emerging Market Bond Index Global (EMBI Global)". JP Morgan, New York, August (1999), p. 16.

KAPETANIOS, GEORGE (2002). "Unit Root Testing Against the Alternative Hypotheses up to $m$ Structural Breaks". Working Paper No. 469, Department of Economics, Queen Mary University of London, p. 17.

LEE, JUNSOO and STRAZICICH, MARK C. (1999). "Minimum LM Unit Root Test with One Structural Break". Department of Economics, Appalachian State University, p. 16.

LUMSDAINE, ROBIN L. and PAPELL, DAVID H.(1997). "Multiple Trend Breaks and the Unit-Root Hypothesis". The Review of Economics and Statistics, Vol. 79, No. 2, pp. 212-218.

MASCAREÑAS, JUAN. (2008).“El Riesgo País”. Finanzas Corporativas, Universidad Complutense de Madrid, p. 28.

MACKINNON, J. G., HAUG,A. A. and MICHELIS, L. (1999). "Numerical distribution functions of likelihood ratio tests for cointegration". Journal of Applied Econometrics, 14, pp. 563-577.

MÁRQUEZ POZOS, JORGE MIGUEL; ISLAS CAMARGO, ALEJANDRO y VENEGAS MARTÍNEZ, FRANCISCO (2003). "Corrientes Internacionales de Capital e Inversión Extranjera de Cartera el caso de México, 1989-1999". El Trimestre Económico, No. 280, Vol. LXX, Octubre-Diciembre, pp. 791-893.

NELSON, C. R., and PLOSSER, C. I. (1982). "Trends and Random Walks in Macroeconomic Time Series: Some Evidence and Implications". Journal of Monetary Economics, 10, pp. 139-162.

NG, SERENA and PERRON, PIERRE(1996). "Lag length Selection and the Construction of Unit Root Tests whit Good Size and Power". Boston College. p.19.

NG, SERENA and PERRON, PIERRE (2001). "Unit Root Tests in ARMA Methods for the Selection of the Truncation Lag". Econometrica, 69, pp. 1519-1554.

PERRON, PIERRE (1989). "The great crash, the oil prices shock, and the unit root hypothesis". Econometrica, vol. 57 num. 6, pp. 1361-1410.

PERRON, PIERRE and VOGELSANG, TIMOTHY J. (1992). "Testing for a Unit Root in a Time Series with a Changing Mean: Corrections and Extensions". Journal of Business \& Economic Statistics, Vol. 10, No. 4, pp. 467-470.

PERRON, P. and RODRÍGUEZ, G. (2003). "GLS Detrending, Efficient Unit Root Tests and Structural Change". Journal of Econometrics 115, pp. 1-27.

PERRON, P. y RODRÍGUEZ, G. (2012). "GLS para eliminar los componentes determinísticos, estadísticos de raíz unitaria eficientes y cambio estructural". Economía Vol. XXXV, N 69, enero-junio 2012, pp. 174-203.

PHILLIPS, P.C.B. y PERRON, PIERRE (1988). "Testing for a Unit Root in Time Series Regression". Biometrika, No. 75, pp. 335-346.

ROSAS CHIMAL, MARIO ALBERTO (2000). Análisis de Corte Estructural en la Inversión de Cartera en México. El Colegio de la Frontera Norte, p. 129. 
SAN-MARTÍN-ALBIZURI, NEREA y RODRÍGUEZ-CASTELLANOS, ARTURO (2011). "La imprevisibilidad de las crisis: un análisis empírico sobre los índices de riesgo país". Innovar 21(39), 161-178.

SARGAN, J. D. and BHARGAVA, A (1983). "Testing for Residuals from Least Squares Regression for Being Generated by the Gaussian Random Walk". Econometrica, 51, pp. 153-174.

SCHUSTER GERSON, GALIA (2005). Determinantes del Riesgo País: Una medición a través de los Swaps de deuda. Mexder. p.79.

SCHWERT G. WILLIAM (1989). "Test for Unit Roots: A Monte Carlo Investigation". Journal of Business \& Economic Statistics, Vol. 7, pp. 147-160.

SCHWERT G. WILLIAM (2011). "Stock Volatility during the Recent Financial Crisis". NBER, num. 16976, p. 32.

STOCK, J.H (1990). "Unit Roots, Structural Breaks and Trends". Handbook of Econometrics, IV, pp. 2740-2841.

ZIVOT, E. and ANDREWS, D.W.K.(1992). "Further Evidence of the Great Crash, the Oil Price Shock and the Unit Root Hypothesis". Journal of Business \& Economic Statistics, Vol. 10, pp. 251-270. 


\section{Anexo Estadístico}

\begin{tabular}{|c|c|c|c|c|c|c|c|c|}
\hline Meses & EMBI & IECT & Meses & EMBI & IECT & Meses & EMBI & IECT \\
\hline ene-98 & 501 & $46,838.74$ & jun-00 & 683 & $68,026.13$ & jul-02 & 915 & $48,411.48$ \\
\hline feb-98 & 460 & $48,098.58$ & jul-00 & 652 & $65,408.17$ & ago-02 & 825 & $49,084.80$ \\
\hline mar-98 & 472 & $53,028.52$ & ago-00 & 627 & $68,992.58$ & sep-02 & 938 & $44,375.19$ \\
\hline abr-98 & 437 & $52,561.46$ & sep-00 & 648 & $64,371.76$ & oct-02 & 818 & $46,797.83$ \\
\hline may-98 & 532 & $44,786.94$ & oct-00 & 731 & $63,122.94$ & nov-02 & 742 & $48,611.79$ \\
\hline jun-98 & 610 & $41,997.98$ & nov-00 & 752 & $56,468.59$ & dic-02 & 724 & $46,028.30$ \\
\hline jul-98 & 543 & $42,489.62$ & dic-00 & 730 & $53,698.39$ & ene-03 & 693 & $44,548.84$ \\
\hline ago-98 & 1377 & $27,655.24$ & ene-01 & 657 & $61,614.55$ & feb-03 & 669 & $43,511.23$ \\
\hline sep-98 & 1243 & $30,859.69$ & feb-01 & 719 & $57,374.22$ & mar-03 & 630 & $44,622.81$ \\
\hline oct-98 & 1058 & $34,094.73$ & mar-01 & 752 & $55,600.81$ & abr-03 & 547 & $49,479.56$ \\
\hline nov-98 & 1059 & $33,969.32$ & abr-01 & 749 & $61,033.64$ & may-03 & 518 & $50,337.13$ \\
\hline dic-98 & 1123 & $34,952.84$ & may-01 & 720 & $66,200.75$ & jun-03 & 517 & $51,977.75$ \\
\hline ene-99 & 1056 & $33,925.28$ & jun-01 & 716 & $62,597.29$ & jul-03 & 503 & $53,842.64$ \\
\hline feb-99 & 1069 & $37,091.07$ & jul-01 & 880 & $58,453.84$ & ago-03 & 502 & $53,128.18$ \\
\hline mar-99 & 919 & $44,009.63$ & ago-01 & 833 & $68,357.07$ & sep-03 & 473 & $53,718.20$ \\
\hline abr-99 & 841 & $52,373.64$ & sep-01 & 959 & $50,136.03$ & oct-03 & 455 & $54,632.78$ \\
\hline may-99 & 910 & $50,230.71$ & oct-01 & 992 & $52,509.29$ & nov-03 & 437 & $57,106.94$ \\
\hline jun-99 & 859 & $53,213.20$ & nov-01 & 995 & $54,515.84$ & dic-03 & 405 & $58,578.14$ \\
\hline jul-99 & 905 & $48,975.16$ & dic-01 & 737 & $56,596.86$ & ene-04 & 413 & $63,972.03$ \\
\hline ago-99 & 919 & $48,173.27$ & sep-00 & 648 & $64,371.76$ & feb-04 & 431 & $67,957.55$ \\
\hline sep-99 & 880 & $48,371.69$ & oct-01 & 992 & $52,509.29$ & mar-04 & 411 & $72,716.37$ \\
\hline oct-99 & 801 & $51,791.36$ & nov-01 & 995 & $54,515.84$ & abr-04 & 452 & $67,579.66$ \\
\hline nov-99 & 757 & $58,312.06$ & dic-01 & 737 & $56,596.86$ & may-04 & 494 & $67,462.91$ \\
\hline dic-99 & 663 & $68,867.29$ & ene-02 & 722 & $64,255.76$ & jun-04 & 481 & $69,619.14$ \\
\hline ene-00 & 695 & $64,854.93$ & feb-02 & 631 & $62,460.11$ & jul-04 & 453 & $65,521.25$ \\
\hline feb-00 & 636 & $74,995.39$ & mar-02 & 581 & $68,407.23$ & ago-04 & 425 & $66,180.74$ \\
\hline mar-00 & 631 & $74,916.21$ & abr-02 & 586 & $65,662.56$ & sep-04 & 409 & $70,537.60$ \\
\hline abr-00 & 673 & $69,698.14$ & may-02 & 623 & $60,450.41$ & oct-04 & 399 & $74,222.84$ \\
\hline may-00 & 737 & $60,688.55$ & jun-02 & 780 & $55,147.56$ & nov-04 & 363 & $75,385.21$ \\
\hline dic-04 & 347 & $80,758.13$ & jun-07 & 181 & $171,985.06$ & dic-09 & 277 & $211,226.18$ \\
\hline ene-05 & 356 & $81,506.50$ & jul-07 & 225 & $165,658.80$ & ene-10 & 309 & $211,525.43$ \\
\hline feb-05 & 333 & $87,717.80$ & ago-07 & 235 & $158,110.77$ & feb-10 & 292 & $212,305.28$ \\
\hline mar-05 & 373 & $80,219.51$ & sep-07 & 214 & $163,084.16$ & mar-10 & 249 & $209,261.98$ \\
\hline abr-05 & 384 & $78,830.12$ & oct-07 & 201 & $168,447.68$ & abr-10 & 258 & $211,146.00$ \\
\hline may-05 & 364 & $83,952.10$ & nov-07 & 261 & $154,773.01$ & may-10 & 317 & $216,140.29$ \\
\hline jun-05 & 297 & $89,010.31$ & dic-07 & 255 & $155,640.57$ & jun-10 & 338 & $213,650.91$ \\
\hline jul-05 & 276 & $96,397.50$ & ene-08 & 287 & $148,692.16$ & jul-10 & 285 & $215,072.00$ \\
\hline ago-05 & 283 & $95,637.64$ & feb-08 & 306 & $158,785.07$ & ago-10 & 299 & $217,510.93$ \\
\hline sep-05 & 235 & $106,158.48$ & mar-08 & 324 & $156,785.89$ & sep-10 & 276 & $197,973.34$ \\
\hline oct-05 & 242 & $104,216.07$ & abr-08 & 283 & $155,928.29$ & oct-10 & 242 & $245,016.08$ \\
\hline nov-05 & 232 & $112,487.53$ & may-08 & 261 & $165,003.77$ & nov-10 & 272 & $209,279.71$ \\
\hline dic-05 & 237 & $116,199.09$ & jun-08 & 308 & $150,743.53$ & dic-10 & 244 & $201,848.65$ \\
\hline ene-06 & 210 & $126,958.75$ & jul-08 & 309 & $147,021.20$ & ene-11 & 270 & $216,281.91$ \\
\hline feb-06 & 187 & $126,883.50$ & sep-08 & 394 & $112,136.39$ & mar-11 & 257 & $262,591.44$ \\
\hline mar-06 & 191 & $127,649.23$ & oct-08 & 619 & $100,958.38$ & $a b r-11$ & 275 & $229,257.10$ \\
\hline
\end{tabular}




\section{Anexo Estadístico (Continuación)}

\begin{tabular}{|c|c|c|c|c|c|c|c|c|}
\hline Meses & EMBI & IECT & Meses & EMBI & IECT & Meses & EMBI & IECT \\
\hline abr-06 & 179 & $135,354.40$ & nov-08 & 707 & $170,327.97$ & may-11 & 285 & $276,037.23$ \\
\hline may-06 & 216 & $122,085.92$ & dic-08 & 689 & $179,976.15$ & jun-11 & 263 & $266,524.74$ \\
\hline jun-06 & 218 & $125,059.66$ & ene-09 & 628 & $185,580.39$ & jul-11 & 275 & $248,154.36$ \\
\hline jul-06 & 197 & $130,805.81$ & feb-09 & 639 & $185,529.70$ & ago-11 & 322 & $233,400.32$ \\
\hline ago-06 & 197 & $132,833.49$ & mar-09 & 637 & $203,299.27$ & sep-11 & 418 & $236,465.34$ \\
\hline sep-06 & 208 & $138,605.75$ & abr-09 & 532 & $220,677.07$ & oct-11 & 351 & $249,802.25$ \\
\hline oct-06 & 194 & $149,978.18$ & may-09 & 459 & $191,579.99$ & nov-11 & 371 & $237,770.67$ \\
\hline nov-06 & 200 & $157,552.85$ & jun-09 & 445 & $186,548.97$ & dic-11 & 374 & $206,722.24$ \\
\hline dic-06 & 171 & $165,703.02$ & jul-09 & 397 & $184,470.19$ & ene-12 & 365 & $249,931.42$ \\
\hline ene-07 & 174 & $159,582.28$ & ago-09 & 381 & $215,630.74$ & & & \\
\hline feb-07 & 187 & $153,768.74$ & sep-08 & 394 & $112,136.39$ & & & \\
\hline mar-07 & 170 & $161,804.76$ & sep-09 & 329 & $264,208.12$ & & & \\
\hline abr-07 & 167 & $168,532.52$ & oct-09 & 323 & $308,222.08$ & & & \\
\hline may-07 & 155 & $172,197.26$ & nov-09 & 326 & $212,122.33$ & & & \\
\hline
\end{tabular}


